\title{
EGFR/JIP-4/JNK2 Signaling Attenuates Cetuximab- Mediated Radiosensitization of Squamous Cell Carcinoma Cells
}

\author{
Iris Eke ${ }^{1}$, Lydia Schneider ${ }^{1}$, Claudia Förster ${ }^{1}$, Daniel Zips ${ }^{1,2}$, Leoni A. Kunz-Schughart ${ }^{1}$, and Nils Cordes ${ }^{1,2}$
}

\begin{abstract}
EGF receptor (EGFR) promotes tumor growth as well as radio- and chemoresistance in various human malignancies including squamous cell carcinomas (SCC). In addition to deactivation of prosurvival signaling, cetuximab-mediated EGFR targeting might concomitantly induce self-attenuating signaling bypasses. Identification of such bypass mechanisms is key to improve the efficacy of targeted approaches. Here, we show great similarity of EGFR signaling and radiation survival in cetuximab-treated SCC cells grown in a more physiologic three-dimensional extracellular matrix and as tumor xenografts in contrast to conventional monolayer cell cultures. Using phosphoproteome arrays, we observed strong induction of JNK2 phosphorylation potentially resulting from cetuximab-inhibited EGFR through c-jun- $\mathrm{NH}_{2}$-kinase (JNK)-interacting protein-4 (JIP-4), which was identified using an immunoprecipitation-mass spectrometric approach. Inhibition of this signaling bypass by JIP-4 or JNK2 knockdown or pharmacologic JNK2 inhibition enhanced cetuximab efficacy and tumor cell radiosensitivity. Our findings add new facets to EGFR signaling and indicate signaling bypass possibilities of cancer cells to improve their survival on cetuximab treatment. By deactivation of cetuximab-self-attenuating JNK2-dependent signaling, the cytotoxicity, and radiosensitizing potential of cetuximab can be augmented. Cancer Res; 73(1); 297-306. (C2012 AACR.
\end{abstract}

\section{Introduction}

The EGF receptor (EGFR) belongs to the ErbB family of transmembrane receptor tyrosine kinases (RTK; ref. 1). Because of increased activity and/or expression in human malignancies and its role in, for example, cell proliferation, migration, and invasion, EGFR emerged as a potent molecular target (2-4). On binding of ligands, such as EGF or amphiregulin, EGFR dimerization results in RTK autophosphorylation and channeling of mitogenic signals predominantly via the Ras-extracellular signal-regulated kinase (ERK), Akt, and c-jun- $\mathrm{NH}_{2}$-kinase (JNK) pathway (1).

Preclinical work showed the effectiveness of pharmacologic inhibitors and antibodies against EGFR in tumor cells from head and neck, lung, colon, and other cancers (3, 5-10). Cetuximab, for example, is approved for use in combination

Authors' Affiliations: ${ }^{1}$ OncoRay - National Center for Radiation Research in Oncology, Medical Faculty Carl Gustav Carus; and ${ }^{2}$ Department of Radiation Oncology, University Hospital and Medical Faculty Carl Gustav Carus, Dresden University of Technology, Dresden, Germany

Note: Supplementary data for this article are available at Cancer Research Online (http://cancerres.aacrjournals.org/).

Corresponding Author: Nils Cordes, OncoRay-National Center for Radiation Research in Oncology, Medical Faculty Carl Gustav Carus, Dresden University of Technology, Fetscherstrasse 74/PF 41, 01307 Dresden, Germany. Phone: 49-0351-458-7401; Fax: 49-0351-458-7311; E-mail:

Nils.Cordes@Oncoray.de

doi: 10.1158/0008-5472.CAN-12-2021

(C)2012 American Association for Cancer Research. with radiotherapy for treatment-naive patients with locally advanced squamous cell carcinoma of the head and neck (HNSCC) and as monotherapy for recurrent or metastatic HNSCC $(11,12)$. These trials showed significantly increased rates of both locoregional control and overall survival.

Despite this clinical success, escape- or efficacy-attenuating mechanisms in response to cetuximab treatment have been widely neglected. Recent studies suggested EGFR ubiquitination, cooperative EGFR-Src family kinase interactions, ErbB2 signaling, and p53 are involved in reduced cetuximab efficacy in colorectal or non-small cell lung cancer cell lines (13-16). As our understanding of EGFR signaling in cancer is still marginal and obviously tumor type-dependent, it is of high clinical relevance to elucidate escape mechanisms of cetuximab in HNSCC by taking into consideration how HNSCC cells grow in vivo. Our own work and work from others have shown that 3dimensional (3D) growth conditions are more physiologically relevant with regard to cell morphology, gene and protein expression patterns, protein-protein interactions, and intracellular signaling as well as response to irradiation and chemotherapeutics relative to conventional $2 \mathrm{D}$ monolayer cell cultures (17-26).

In the present study, we sought to elucidate the nature of EGFR signaling in more detail and evaluate whether cetuximab mediates differential cytotoxicity and radiosensitization in squamous cell carcinoma (SCC) cells grown in 3D versus 2D. In a systematic comparison of different growth conditions, we show significant similarity of EGFR, ERK1/2, and Akt phosphorylation in SCC cells grown in 3D extracellular matrix 
(ECM) and as tumor xenografts in contrast to conventional monolayer cell cultures. In line with in vivo studies assessing cetuximab plus radiotherapy $(7,9)$, cetuximab radiosensitized cells in a 3D, but not $2 \mathrm{D}$, environment. Using phosphoproteome arrays, we observed strong induction of JNK2 phosphorylation potentially resulting from an interaction between cetuximab-inhibited EGFR and JNK-interacting protein-4 (JIP-4), which was identified in an immunoprecipitation-mass spectrometric approach. Inhibition of this signaling bypass by JIP-4 or JNK2 knockdown or pharmacologic JNK2 inhibition enhanced cetuximab efficacy and tumor cell radiosensitivity.

\section{Materials and Methods}

Please see Supplementary Experimental Procedures for the following sections: "Measurement of apoptosis" and "Immunoprecipitation of EGFR in untreated cells."

\section{Antibodies}

Antibodies used for Western blotting include EGFR Y1173, EGFR Y1068, JNK T183/Y185 (Invitrogen), EGFR, Akt, Akt S473, Akt T308, ERK1/2, ERK1/2 T202/Y204, MEK1/2, MEK1/2 S217/
221, JNK, c-Jun, c-Jun S63, JIP-4, caspase-3, phosphotyrosine, phosphothreonine (Cell Signaling), $\beta$-actin (Sigma), horseradish peroxidase-conjugated donkey anti-rabbit, and sheep antimouse antibodies (Amersham). Antibodies for immunofluorescence staining include EGFR (Abcam), JIP-4, c-Jun S63, c-Jun (Cell Signaling), Alexa594 phalloidin, Alexa488 anti-rabbit, and Alexa594 anti-mouse antibodies (Invitrogen). Antibodies for immunoprecipitation include EGFR (Invitrogen) and mouse immunoglobulin G (IgG; Santa Cruz Biotechnology). Antibodies were purchased as indicated.

\section{D and 3D cell culture}

A431 and FaDu cells were purchased from the American Type Culture Collection. The SAS, UTSCC14, and UTSCC5 cells were a generous gift from Dr. R. Grenman (Turku University Central Hospital, Turku, Finland). Asynchronously and exponentially growing cells were used in all experiments. In both 2D and 3D cell-culture conditions, cells were cultured in Dulbecco's Modified Eagle's Medium (DMEM) containing GlutaMAX-I supplemented with $10 \%$ fetal calf serum (except for experiments shown in Supplementary Fig. S1, which were conducted under serum starvation) and $1 \%$ nonessential amino

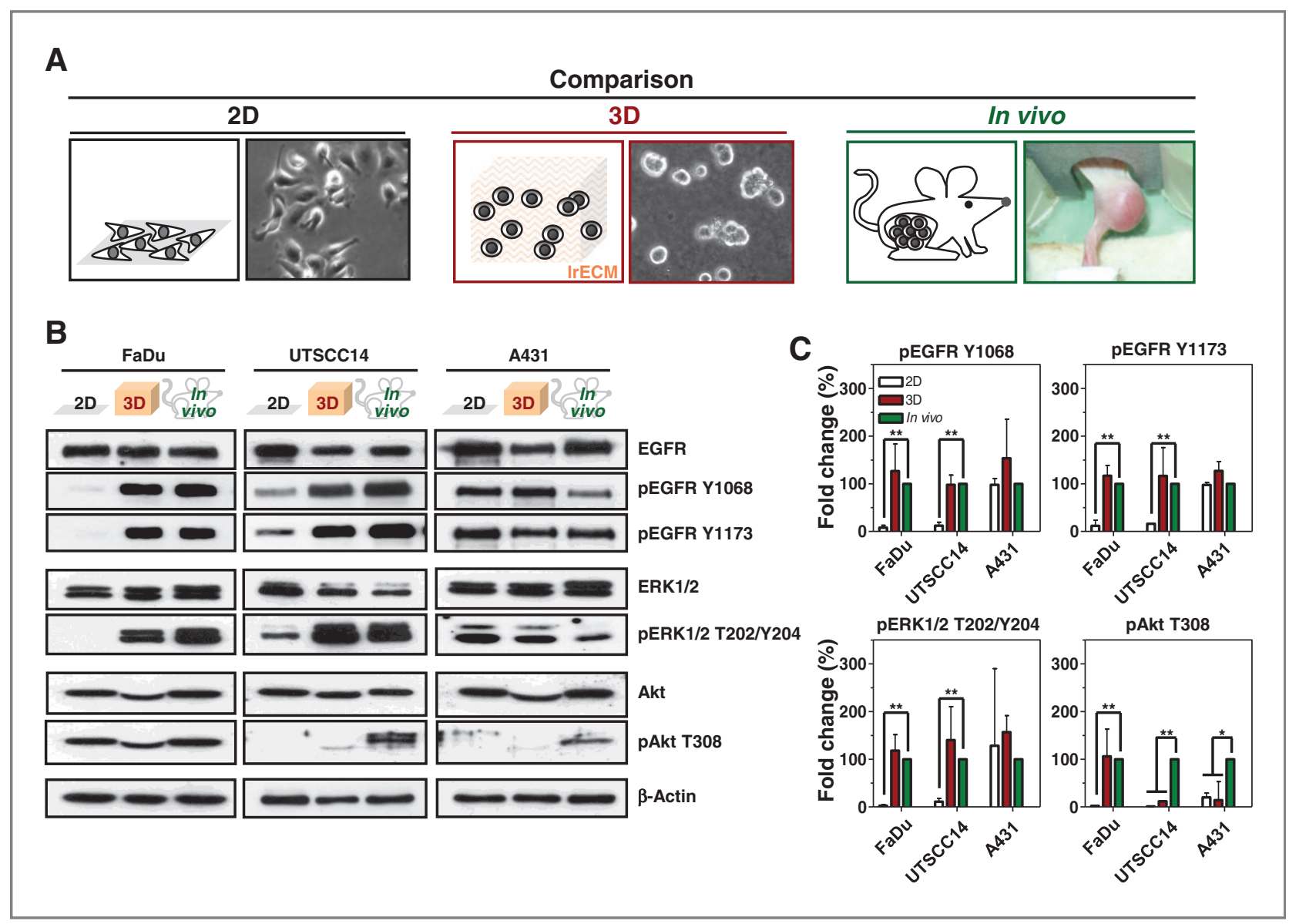

Figure 1. Phosphorylation of EGFR and downstream proteins of SCC cells is similar in 3D cell cultures and SCC xenograft tumors. A, schematic and images of cells cultured in 2D or 3D and as tumor xenograft (in vivo). B, Western blot analysis on whole-cell lysates from 2D and 3D SCC cell cultures and homogenates of tumors grown on nude mice and detection of EGFR and associated signaling molecules. Representative images are shown. $\beta$-Actin served as loading control. $\mathrm{C}$, fold change of protein phosphorylation shown in $\mathrm{B}$ upon normalization to total protein expression (mean $\pm \mathrm{SD} ; n=2 ; t$ test; *, $P<0.05$; ${ }^{* *}, P<0.01$ ). 
acids (PAA) at $37^{\circ} \mathrm{C}$ in a humidified atmosphere containing $7 \%$ $\mathrm{CO}_{2}$. Conventional tissue culture plates (BD Biosciences) were used for 2D cell culture. For 3D cell culture, plates were coated with $1 \%$ agarose (Sigma) to prevent cell attachment to the bottom of the well. Cultrex 3D Culture Matrix (IrECM; Trevigen) was added to the cell-culture medium to obtain a final concentration of $0.5 \mathrm{mg} / \mathrm{mL}$. Thereafter, 3D cultured cells were covered with supplemented DMEM.

\section{Radiation exposure}

Irradiation was delivered at room temperature using single doses of $200 \mathrm{kV} \mathrm{X-rays} \mathrm{(Yxlon} \mathrm{Y.TU} \mathrm{320;} \mathrm{Yxlon)} \mathrm{filtered}$ with $0.5-\mathrm{mm} \mathrm{Cu}$. The absorbed dose was measured using a Duplex dosimeter (PTW). The dose-rate was approximately $1.3 \mathrm{~Gy} / \mathrm{min}$ at $20 \mathrm{~mA}$, and applied doses ranged from 0 to $6 \mathrm{~Gy}$.

\section{D colony formation assay}

The $2 \mathrm{D}$ colony formation assay was conducted as published previously (27). Asynchronously and exponentially growing cells were plated as single cells in 6-well cell-culture plates. After 24 hours, cetuximab was added to the medium to final concentrations of 0 to $5 \mu \mathrm{g} / \mathrm{mL}$. After 24 hours, cells were either left unirradiated or were irradiated (2-6 Gy) and then cultured for 9 days (A431 and UTSCC14) or 11 days (FaDu). Cetuximab remained in the supernatant for the entire culture period. After fixation with $80 \%$ ethanol and Coomassie staining (Merck), cell colonies with more than 50 cells were counted microscopically (Fig. 2A). Plating efficiencies were calculated as follows: number of colonies formed/number of cells plated. Surviving fractions (SF) were calculated as follows: numbers of colonies formed/ (numbers of cells plated (irradiated) $\times$ plating efficiency (unirradiated). Each point on survival curves represents the mean surviving fraction from at least 3 independent experiments.

\section{D colony formation assay}

Clonogenic survival under 3D conditions was determined in a 3D colony formation assay as published previously $(26,27)$. Asynchronously and exponentially growing single cells were mixed with $0.5 \mathrm{mg} / \mathrm{mL}$ lrECM and placed in 96-well plates. LrECM was covered with cell-culture medium. After 24 hours, cetuximab was added to the medium to final concentrations of 0 to $5 \mu \mathrm{g} / \mathrm{mL}$. After 24 hours, irradiation ( $0-6 \mathrm{~Gy}$ ) was carried out. Cetuximab remained in the cell-culture medium for the entire growth period. Incubation of cells with the JNK inhibitor SP600125 (Santa Cruz Biotechnology) was carried out for 26.5 hours, whereby the inhibitor was added 30 minutes before cetuximab and was withdrawn 2 hours after irradiation by washing 5 times using DMEM. Cells were cultured for 9 days (A431 and UTSCC14), 11 days (FaDu), or 14 days (UTSCC5). Cell clusters (with the minimum size of a cell cluster containing

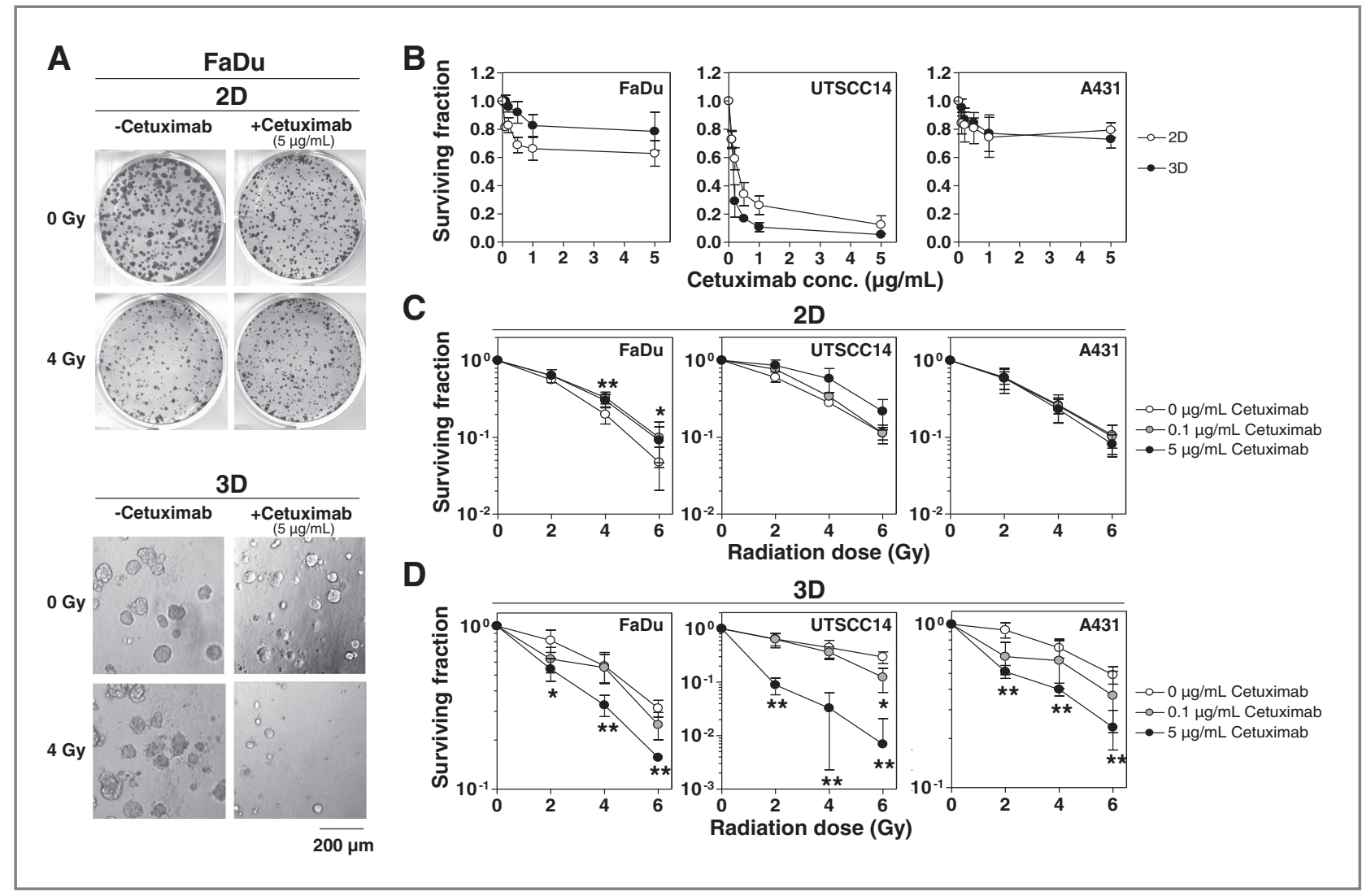

Figure 2. Cytotoxicity and radiosensitizing potential of cetuximab in $2 \mathrm{D}$ and $3 \mathrm{D}$ SCC cell cultures. A, representative images of cell colonies. B, clonogenic survival of SCC cells treated with cetuximab $(5 \mu \mathrm{g} / \mathrm{mL})$. Results show mean $\pm \operatorname{SD}(n=3)$. C and D, colony formation of $2 \mathrm{D}$ and $3 \mathrm{D}$ SCC cell cultures treated with cetuximab for 24 hours before irradiation ( $0-6$ Gy single X-ray doses). Results show mean $\pm \operatorname{SD}\left(n=3 ; t\right.$ test; $\left.{ }^{*}, P<0.05 ;{ }^{* *}, P<0.01\right)$. 
50 cells) were counted microscopically without fixation or staining (see Fig. 2A). Plating efficiencies and surviving fractions were calculated as described earlier. Synergism was calculated as recently published (28) using the following formula: $S=r(a, b)-r\left(a_{0}, b\right) \times r\left(a, b_{0}\right)$.

\section{Total protein extracts and Western blotting}

Asynchronously and exponentially growing 2D cell cultures were rinsed with ice-cold $1 \times$ PBS before harvesting total proteins by scraping with modified radioimmunoprecipitation assay (RIPA) buffer [ $50 \mathrm{mmol} / \mathrm{L}$ Tris- $\mathrm{HCl}$ (pH 7.4), $1 \%$ Nonidet$\mathrm{P} 40,0.25 \%$ sodium deoxycholate, $150 \mathrm{mmol} / \mathrm{L} \mathrm{NaCl}, 1 \mathrm{mmol} / \mathrm{L}$ EDTA, complete protease inhibitor cocktail (Roche), $1 \mathrm{mmol} / \mathrm{L}$ $\mathrm{NaVO} 4$, and $2 \mathrm{mmol} / \mathrm{L} \mathrm{NaF}]$. In 3D, asynchronously and exponentially growing cells were lysed within the IrECM using modified RIPA buffer. Homogenization of lysates was accomplished by 4 passages through a 25-gauge needle followed by centrifugation. Samples were stored at $-80^{\circ} \mathrm{C}$. Protein concentration of $2 \mathrm{D}$ lysates was measured using the BCA assay (Thermo Scientific). SDS-PAGE, transfer of proteins onto nitrocellulose membranes (Schleicher und Schuell), probing, and detection of specific proteins with indicated antibodies and SuperSignal West Dura Extended Duration Substrate (Thermo Scientific) was carried out as described previously (27).

\section{siRNA transfection}

MEK1 siRNA (sequence: 5'-GGAGCUAGAGCUUGAUGAGtt- $3^{\prime}$ ), JNK2 siRNA (sequence: 5 -GGGAUUGUUUGUGCUGCAUtt- $3^{\prime}$ ), EGFR siRNA (sequence: $5^{\prime}$-GGCACGAGUAACAAGCUCAtt- $3^{\prime}$ ), and JIP-4 siRNA (sequence: $5^{\prime}$-GCAGUAUCGUCAGGUAAAAtt- $3^{\prime}$ ) were obtained from Applied Biosystems. The nonspecific control siRNA (sequence: $5^{\prime}$ GCAGCUAUAUGAAUGUUGUtt- $3^{\prime}$ ) was from Eurofins MWG Operon. siRNA transfection was conducted as published (17). Twenty-four hours after delivery of $20 \mathrm{nmol} / \mathrm{L}$ siRNA using oligofectamine (Invitrogen), cells were plated on 2D cell-culture plastic or embedded in 3D lrECM. A 1- or 24hour incubation with cetuximab $(0-5 \mu \mathrm{g} / \mathrm{mL})$ was followed by irradiation. Colony formation assays and Western blotting were carried out. Efficient MEK1, JNK2, EGFR, and JIP-4 knockdown was confirmed by Western blotting.

\footnotetext{
3D immunofluorescence staining

Immunofluorescence stainings of 3D cultured cells were carried out as recently published (8). Three-dimensionally cultured cells were treated with cetuximab $(0-5 \mu \mathrm{g} / \mathrm{mL})$ for 1 hour and then fixed with $3 \%$ formaldehyde/PBS in IrECM for 15 minutes. After transfer to a centrifugation tube, cells were permeabilized with $0.25 \%$ Triton X-100/PBS for 10 minutes, washed with PBS, and blocked with $1 \%$ BSA/PBS for 30 minutes. Staining of c-Jun, c-Jun S63, EGFR, and JIP-4 was carried out with specific antibodies for 2 hours. After washing with $1 \times$ PBS, secondary antibodies and/or Alexa594 phalloidin were added for 1 hour. Cells were washed and transferred onto microscopic slides before covering with Vectashield/DAPI mounting medium (Alexis) and cover slip. Images were acquired using a confocal laser scanning microscope LSM 510meta (Zeiss). Colocalization was measured using the LSM510 software (Zeiss).
}

\section{Animal experiments}

The experiments were carried out using 7- to 14-week-old male and female NMRI (nu/nu) mice obtained from the pathogen-free animal breeding facility (Experimental Center, Medical Faculty, Technische Universität Dresden, Germany). Animal facilities and experiments were approved according to the institutional guidelines and the German animal welfare regulations. For the experiments, small tumor pieces were transplanted subcutaneously into the right hind-leg as published previously $(7,26,27)$. At a tumor size of $7 \mathrm{~mm}$ in diameter, mice were treated with $1 \mathrm{mg}$ cetuximab (Erbitux) intravenously in a volume of $0.2 \mathrm{~mL}$. Please see Supplementary Experimental Procedures for detailed description.

\section{Phospho-specific protein microarray analysis}

The Phospho Explorer Antibody Microarray was conducted by Full Moon BioSystems Inc. Whole-cell lysates from 2D and 3D FaDu cell cultures treated with cetuximab $(5 \mu \mathrm{g} / \mathrm{mL}$; or untreated controls) were harvested after 1 hour using Protein Extraction Buffer (Full Moon BioSystems Inc.) and transferred to Full Moon BioSystems Inc., on dry ice. The array consists of 1,318 phospho-specific antibodies. In brief, proteins were labeled with biotin and placed on preblocked microarray slides. After washing, detection of total and phosphorylated proteins was conducted using Cy3-conjugated streptavidin. Expression of phosphorylated proteins was normalized to corresponding total protein expression. Fold change was calculated as follows: phosphorylation of cetuximab-treated cells/ phosphorylation of untreated cells. This experiment was carried out once. Where indicated, protein phosphorylation data were confirmed by Western blotting.

\section{Immunoprecipitation after cetuximab treatment}

Cetuximab-treated $(5 \mu \mathrm{g} / \mathrm{mL})$ or untreated 3D grown cells were lysed with cell lysis buffer (Cell Signaling) supplemented with complete protease inhibitor cocktail as described $(26,27)$. Protein-G-Agarose beads (Sigma) were incubated with nonspecific mouse IgG antibody overnight. Protein lysates were added to the beads and incubated overnight. Cetuximabbound EGFR was pulled down by interaction of cetuximab with the beads, and not by the nonspecific mouse IgG. After washing of the beads, SDS-PAGE and Western blotting were carried out as described earlier.

Immunoprecipitation and sequential mass spectrometry

Three-dimensionally grown cells were lysed with cell lysis buffer (Cell Signaling) supplemented with complete protease inhibitor cocktail (Roche). Protein-G-Agarose beads (Sigma) were incubated with EGFR antibody or nonspecific mouse IgG antibody overnight. Protein lysates were added to the beads and incubated overnight. After SDS-PAGE, gel fixation, and Coomassie (Merck) staining, mass spectrometric analysis was carried out at the Max Planck Institute of Molecular Cell Biology and Genetics Mass Spectrometry (MPI-CBG MS) Facility (Dresden, Germany) as published $(26,27)$. Proteins were first in-gel digested with trypsin as described in ref. 29 and the liquid chromatography/tandem mass spectrometric (LC/MS-MS) analysis of extracted peptide mixtures was conducted on an 
Ultimate nanoLC systems (Dionex) interfaced online to a LTQ Orbitrap hybrid mass spectrometer (Thermo-Fisher Scientific) via a robotic nanoflow ion source TriVersa (Advion BioSciences Ltd.) as described in ref. (29). Acquired spectra were searched against human international protein index (IPI) protein databases. Spectra were matched by MASCOT software (Matrix Science, v.2.2.0) under the following settings: mass tolerance was set as $\pm 10 \mathrm{ppm}$ for precursor and $\pm 0.5 \mathrm{Da}$ for fragmentation spectra; variable modifications: propionamide (C), $N$-acetylation (Protein N-terminus), oxidation (M); enzyme: trypsin; and 1 missed cleavage allowed. The result was evaluated by Scaffold software (Proteome Software) under the following settings: minimal number of peptides was 2; minimal peptide and protein probabilities were $95 \%$ and $99 \%$, respectively.

\section{Data analysis}

Means \pm SD of at least 3 independent experiments were calculated with reference to untreated controls defined in a 1.0 scale. To test statistical significance, Student $t$ test was conducted using Microsoft Excel 2003. Results were considered statistically significant if a $P$ value of less than 0.05 was reached. Densitometry of Western blot analyses was conducted with ImageJ analysis software (http://rsbweb.nih. gov/ij).

\section{Results}

\section{Growth conditions severely impact on EGFR phosphorylation and signaling}

We first characterized and compared EGFR expression and phosphorylation of 3 SCC cell lines in 3D IrECM versus conventional monolayer cultures versus tumor xenografts (Fig. 1A). Intriguingly, patterns of EGFR expression and EGFR tyrosine (Y)1068 and Y1173 as well as ERK1/2 threonine (T) 202/Y204 phosphorylation showed great similarity in FaDu and UTSCC14 cells grown in 3D lrECM and in vivo (Fig. 1B and C). Akt T308 phosphorylation was higher in 3D than in 2D, but only matched with in vivo levels in FaDu cells (Fig. $1 \mathrm{~B}$ and C). The extreme levels of EGFR phosphorylation in A431 cells might be causative for only small detectable differences of proteins of interest under the different growth conditions (Fig. 1B and C). To our knowledge, this is the first comparative analysis of EGFR signaling in HNSCC cells growing in $3 \mathrm{D}$, in vivo, or as a

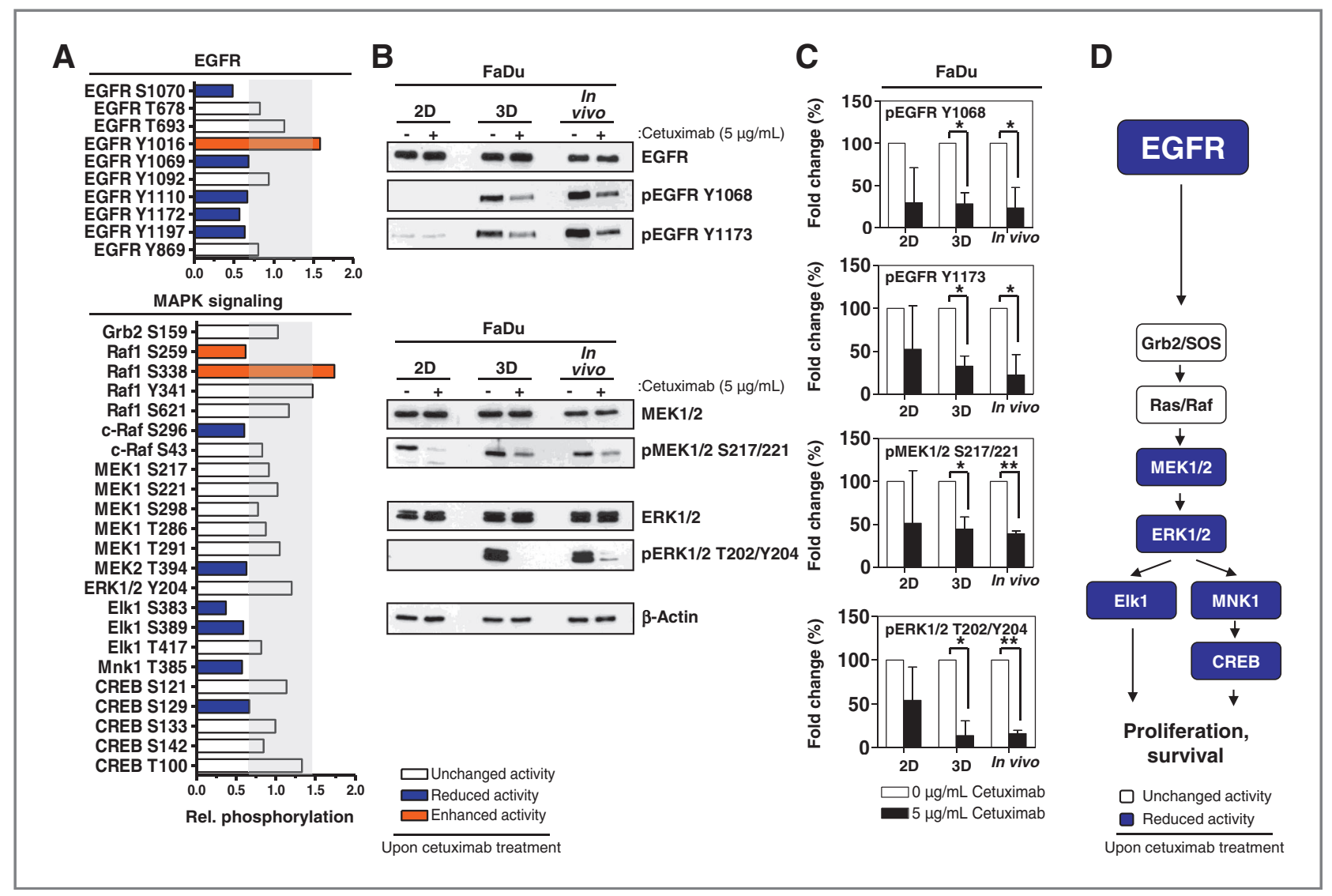

Figure 3. Phosphoproteome array data of cetuximab-treated 3D FaDu cell cultures (EGFR and MAPK signaling). A, the Phospho Explorer Antibody Array (details in Materials and Methods) was used to screen total cell lysates from untreated and cetuximab-treated 3D FaDu cell cultures $(n=1)$. Data show fold change of indicated phosphoproteins upon cetuximab treatment after normalization to total protein expression. Gray-colored area indicates the defined induction/reduction boundaries $(\leq 67 \% / \geq 150 \%)$. B, confirmatory Western blot analyses of $2 \mathrm{D}$ and $3 \mathrm{D}$ cell cultures and tumor xenografts after treatment with cetuximab. C, densitometric analysis of Western blot analyses shown in B. Phosphorylation was normalized to total protein expression (mean $\pm \mathrm{SD} ; n=2 ; t$ test; ${ }^{*}, P<0.05 ;{ }^{* \star} P,<0.01$ ). D, schematic of activated and deactivated proteins of the MAPK signaling pathway upon cetuximab treatment (according to www.phosphosite.org). 
monolayer, providing strong support for 3D lrECM-based cell cultures as correlates to in vivo growth conditions.

Cytotoxicity and radiosensitizing potential of cetuximab

The inhibitory potential of cetuximab under serum starvation and subsequent EGF stimulation was conducted. EGF exposure resulted in enhanced EGFR Y1173 and Y1068 phosphorylation in FaDu and UTSCC14 cells (Supplementary Fig. S1). Next, we analyzed the effects of cetuximab on clonogenic survival of 3D- and 2D-grown SCC cell lines and found great intercell line variances independently of 3D or 2D culture settings (Fig. 2A and B; Supplementary Table S1). In contrast to monolayer cell cultures (Fig. 2A and C), 3D lrECM cell cultures were significantly $(P<0.01)$ and concentration-dependently radiosensitized by cetuximab (Fig. $2 \mathrm{~A}$ and D), whereas apoptosis induction was negligible in this context (Supplementary Figs. S2 and S3). From these results, we conclude cetuximab efficacy alone to be independent of growth conditions in contrast to combined cetuximab/irradiation treatment regimens in SCC cell lines.

\section{Phosphoproteome modifications after cetuximab- mediated EGFR inhibition}

Next, we sought to identify the modifications of the intracellular signaling network resulting from cetuximab treatment by using a phosphoproteome array technique. This array contains 1,318 well-characterized phospho-specific antibodies.
EGFR and ERK1/2-, Akt-, and JNK-related signaling pathways were selected from the complete data set (data not shown). Only proteins with modified phosphorylation of $67 \%$ or less and $150 \%$ or more from cetuximab-treated 3D FaDu cell cultures were listed according to pathway after normalization to untreated controls (Figs. 3A, 4A). In general, the data showed reduced phosphorylation at kinase-stimulating sites as well as increased phosphorylation at kinase-inhibitory sites in response to cetuximab (Figs. 3A, 4A). Detection of total phosphotyrosine and phosphothreonine levels supported this observation (Supplementary Fig. S4). For EGFR, we found reduced phosphorylation at 5 of 10 examined EGFR sites, that is, Y1068/1069 and Y1172/1173; borderline changes at 4 sites; and induction at EGFR Y1016 (Fig. 3A). Selective confirmatory Western blotting analysis of basal and cetuximab-related EGFR phosphorylation patterns showed approximately $70 \%$ reduction in EGFR phosphorylation at particular sites independent from growth conditions; however, for 3D cell culture and tumor xenograft, the reduction was significant (Fig. 3B and C). Moreover, inhibition of EGFR resulted in decreased phosphorylation levels of downstream mitogen-activated protein kinase (MAPK) signaling pathways including MAPK kinase [MAP-ERK kinase (MEK); $\sim 45 \%$ reduction in Western blot analysis], ERK1/2 ( $\sim 80 \%$ reduction in Western blot analysis), $\mathrm{E}$ twenty-six-like transcription factor 1 (Elk1), MAPK-interacting kinase 1 (MNK1), and cAMP response element-binding protein (CREB; Fig. 3). Interestingly, changes in growth factor

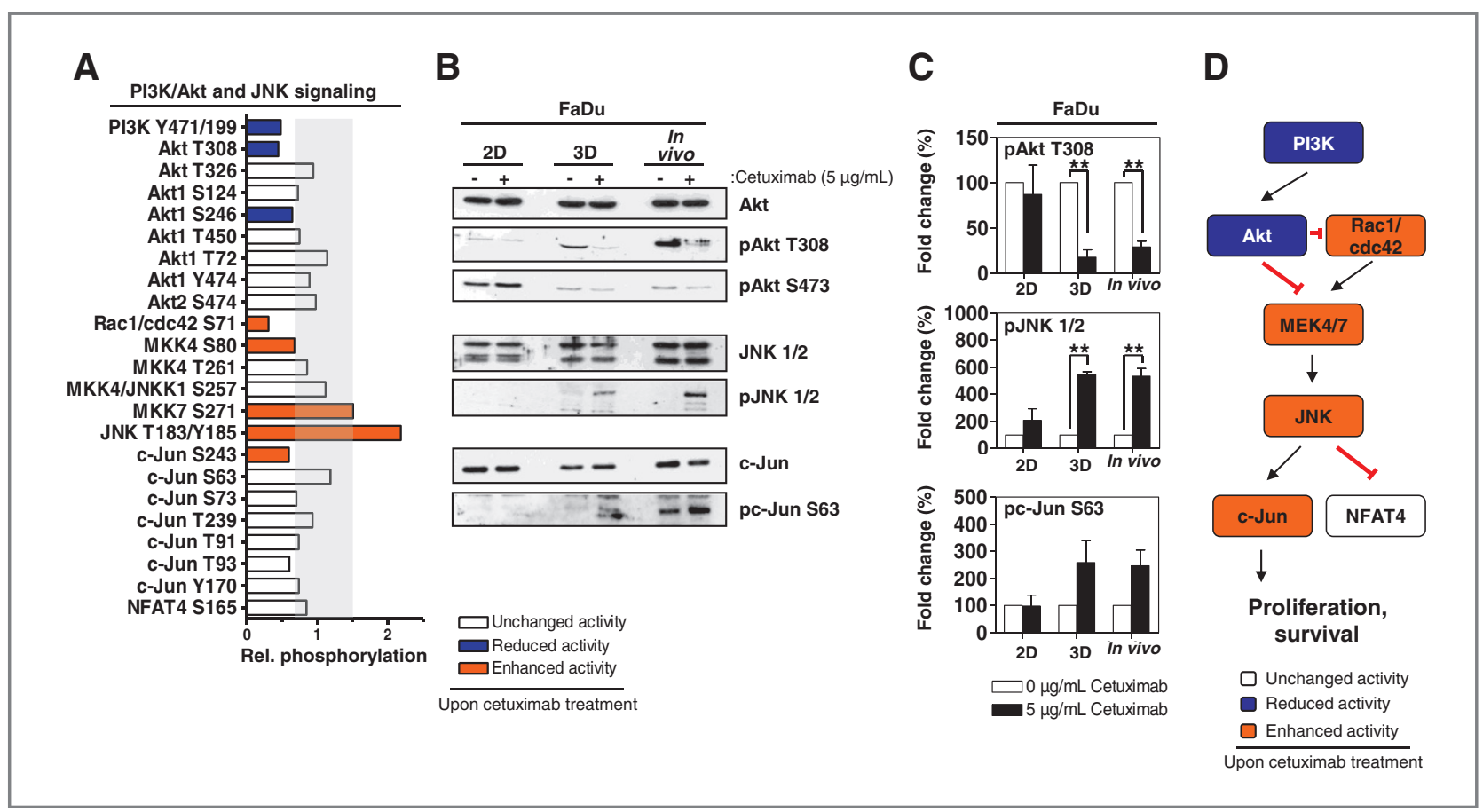

Figure 4. Phosphoproteome array data of cetuximab-treated 3D FaDu cell cultures (PI3K/Akt and JNK signaling). A, the Phospho Explorer Antibody Array (details in Materials and Methods) was used to screen total cell lysates from untreated and cetuximab-treated 3D FaDu cell cultures $(n=1)$. Data show fold change of indicated phosphoproteins upon cetuximab treatment after normalization to total protein expression. Gray-colored area indicates the defined induction/reduction boundaries $(\leq 67 \% / \geq 150 \%)$. B, confirmatory Western blot analyses of $2 \mathrm{D}$ and $3 \mathrm{D}$ cell cultures and tumor xenografts after treatment with cetuximab. C, densitometric analysis of Western blot analyses shown in B. Phosphorylation was normalized to total protein expression (mean \pm SD; $n=2 ; t$ test; ${ }^{*}, P<0.05 ;{ }^{* \star}, P<0.01$ ). D, schematic of activated or deactivated proteins of the PI3K/Akt and JNK signaling pathway upon cetuximab treatment (according to www.phosphosite.org). 
receptor-bound protein 2 (Grb2), Ras, and Raf were not observed. EGFR blocking also led to significant dephosphorylation of phosphoinositide 3-kinase (PI3K) and Akt in 3D and in vivo by $80 \%$ and $70 \%$, respectively, in contrast to $2 \mathrm{D}$ (Fig. 4; according to www.phosphosite.org). Besides kinase-deactivating phosphorylations, we detected hyperphosphorylation of Rac-MEK4/7-JNK-c-Jun signaling components in both 3D HNSCC cell cultures and tumor xenografts upon cetuximab treatment relative to untreated controls (Figs. 4 and 5A-C). Intriguingly, phosphorylation of JNK1/2 and c-Jun S63 was highly significantly induced upon cetuximab treatment by approximately $600 \%$ and $250 \%$, respectively, in $3 \mathrm{D}$ and in vivo as compared with 2D (Fig. 4B and C). The presented phosphoproteome data in 3D lrECM HNSCC cell cultures suggest cetuximab causes a distinct EGFR dephosphorylation pattern that translates into selective inhibition of proteins associated with the MEK1/2-ERK1/2 and PI3K/Akt signaling cascade. Of specific interest is the similarity of signal transduction events in these 2 cascades in HNSCC cells grown in 3D lrECM and as xenograft tumors. These findings support our supposition that
3D lrECM-based cell cultures better reflect in vivo growth conditions compared with 2D monolayers.

\section{JNK2 depletion enhances cetuximab-induced radiosensitization}

Because of the context dependency of JNK with regard to antiand prosurvival effects, we next investigated whether the cetuximab-induced JNK2 hyperphosphorylation modifies cetuximab efficacy (Figs. 4 and 5A). JNK2 targeting using siRNA or the pharmacologic inhibitor SP600125 resulted in significant cytotoxicity in 3D FaDu cell cultures (Fig. 5B and C; Supplementary Fig. S5). While JNK2 depletion significantly sensitized 3D-grown FaDu cells to X-rays, combined cetuximab plus JNK2 depletion showed additive effects in contrast to cetuximab plus MEK1 knockdown (Fig. 5B; Supplementary Table S3). Similar results were obtained on SP600125 treatment (Fig. 5C). EGFR knockdown caused higher radiosensitization than cetuximab alone and remained unchanged by additional cetuximab administration (Fig. 5D). Notably, EGFR depletion prevented cetuximabinduced JNK2 hyperphosphorylation, and JNK inhibition

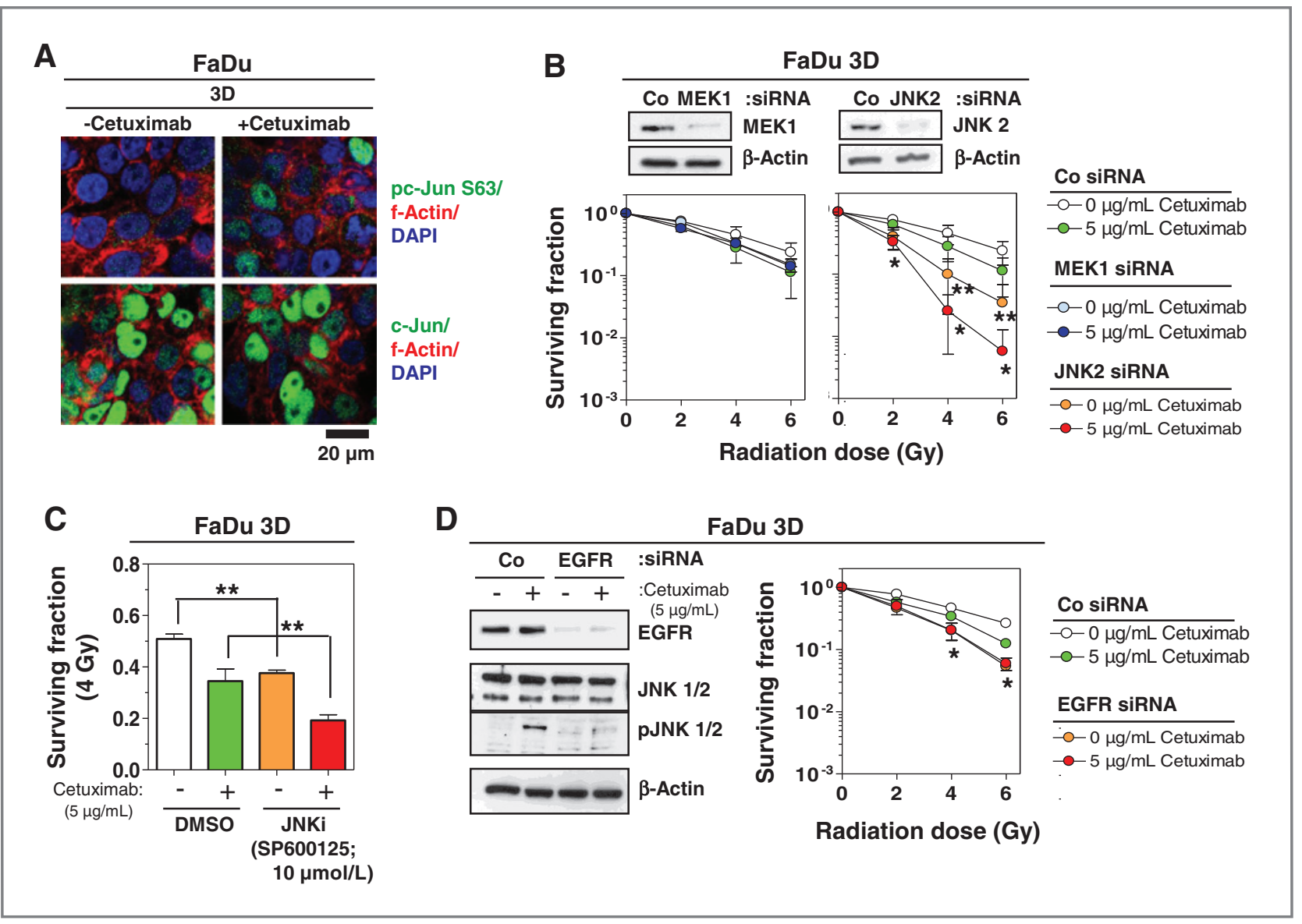

Figure 5. Effects of combined cetuximab plus JNK2 knockdown on 3D SCC cell-culture radiosensitivity. A, immunofluorescence staining and confocal laser scanning microscopy of total and phosphorylated c-Jun S63 upon cetuximab. B, clonogenic radiation survival of 3D-grown MEK1 and JNK2 knockdown cell cultures additionally treated with cetuximab. Results show mean $\pm \mathrm{SD}(n=3 ; t$ test). C, colony formation of 4 to Gy irradiated $3 \mathrm{D}$ grown cell cultures pretreated with JNKi $(10 \mu \mathrm{mol} / \mathrm{L})$ for 30 minutes before 1 -hour cetuximab treatment (mean $\pm \mathrm{SD} ; n=3 ; t$ test). D, Western blot analysis and clonogenic survival of 3D-grown cells upon treatment with cetuximab under EGFR knockdown. Results show mean \pm SD ( $n=3$; $t$ test). Co siRNA, nonspecific siRNA control $\left(*, P<0.05 ;{ }^{* *}, P<0.01\right)$. 


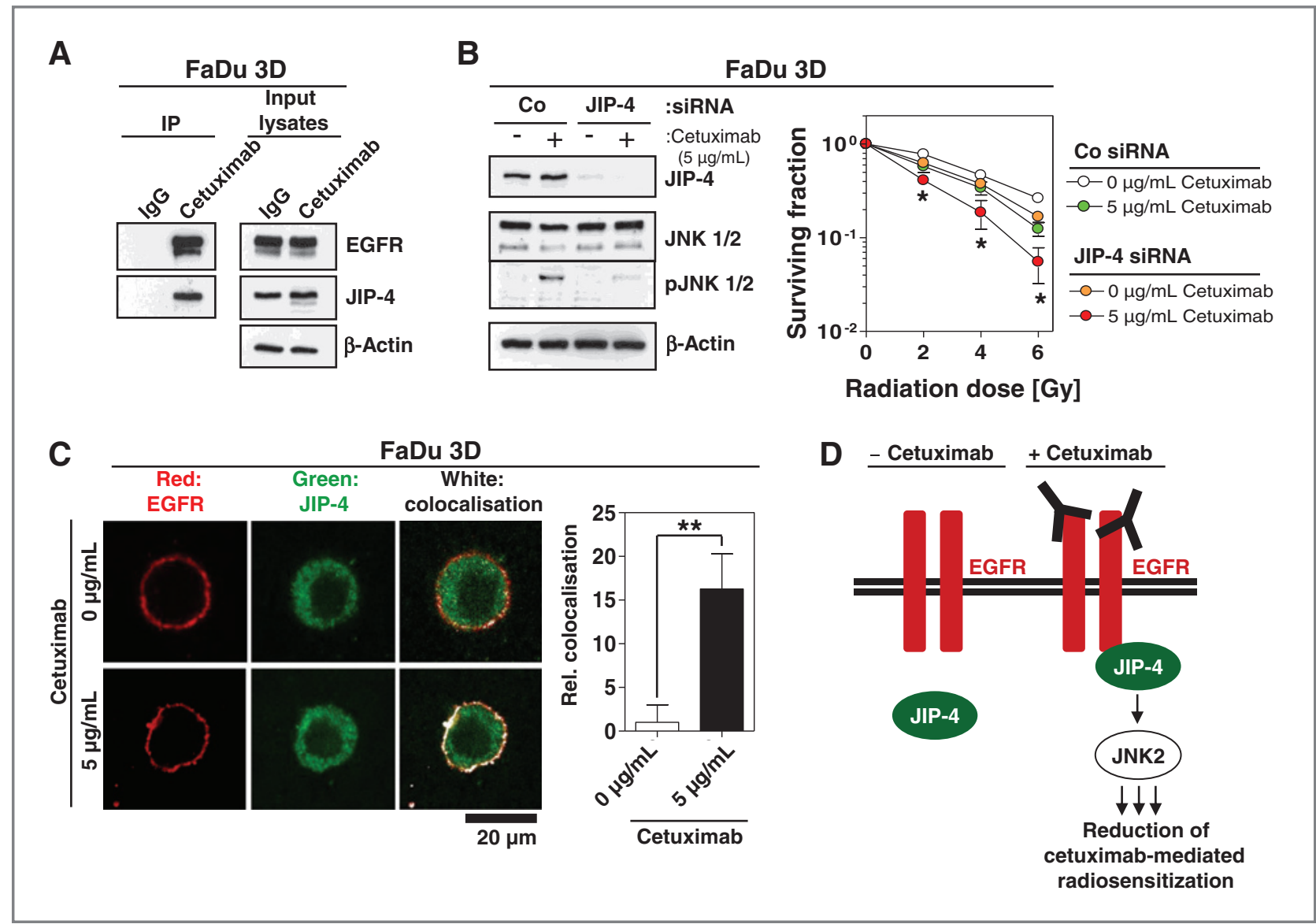

Figure 6. JIP-4 is recruited to EGFR upon treatment with cetuximab and facilitates JNK2 phosphorylation. A, immunoprecipitation (nonspecific IgG control and input lysates used for immunoprecipitation) in cetuximab-treated and untreated 3D IrECM FaDu cell cultures (see also Supplementary Table S1). B, Western blot analysis and clonogenic survival of cetuximab-treated, JIP-4 knockdown 3D cell cultures. Results show mean \pm SD ( $n=3 ; t$ test). C, immunofluorescence staining of EGFR and JIP-4 in 3D grown cells with and without cetuximab treatment. Colocalization was determined with LSM510 software. Results show mean $\pm \mathrm{SD}(n=3 ; t$ test). D, schematic of how cetuximab results in JNK2 activation via recruitment of JIP-4 to EGFR. Co siRNA, nonspecific siRNA control $\left(*, P<0.05 ;{ }^{* *}, P<0.01\right)$.

abrogated cetuximab-dependent activation of c-Jun (Fig. 5D; Supplementary Fig. S6). In cetuximab-resistant UTSCC5 cells, supportive data were generated delineating JNK2 to be key for radiosensitization by cetuximab (Supplementary Fig. S7 and Supplementary Table S3). These observations show a novel link between EGFR and JNK2 signaling and suggest this interaction plays a critical role in the self-attenuating process that reduces the radiosensitizing potential of cetuximab.

\section{JIP-4 connects EGFR to JNK2}

By carrying out mass spectrometry on EGFR-immunoprecipitates, we identified a variety of interacting proteins (Supplementary Table S2). The most potent candidate found in these immunoprecipitates from cetuximab-treated $3 \mathrm{D} \mathrm{FaDu}$ cell cultures that connects EGFR to JNK signaling was JIP-4 (Fig. 6A; Supplementary Fig. S8). The radiosensitization by JIP4 depletion was superimposable to that of cetuximab, and cetuximab-induced JNK2 hyperphosphorylation was prevented (Fig. 6B; Supplementary Table S3). An enhanced recruitment of JIP-4 to cetuximab-inhibited EGFR was visualized by immunofluorescence staining and colocalization measure- ment in 3D FaDu cell cultures as compared with controls (Fig. 6C). These data show a functional role of JNK2 in the radiation survival response of 3D lrECM grown cells and suggest JNK2 activation under cetuximab-dependent EGFR inhibition to be transduced by interaction of EGFR with JIP-4 (Fig. 6D).

\section{Discussion}

Understanding EGFR signaling in cancer is essential for treatment optimization and individualization. In addition to a plethora of novel molecular biology technologies, 3D cell cultures have been postulated to better reflect in vivo growth condition and, therefore, might be highly valuable to support this endeavor. Here, we present data showing great similarity in EGFR signal transduction events between 3D IrECM HNSCC cell cultures and HNSCC tumor xenografts in contrast to monolayer cell cultures. Effective EGFR inhibition by cetuximab elicited concentration-dependent cytotoxicity and radiosensitization. In our phosphoproteome analyses, we observed a strong induction of JNK2 phosphorylation potentially resulting from cetuximab-inhibited EGFR. Suppressing bypass signaling 
via JIP-4 or JNK2 inhibition enhanced cetuximab efficacy and tumor cell radiosensitivity. These findings add new facets to EGFR signaling with particular focus on resistance pathways, which limit tumor cell eradication and radiosensitization.

As emphasized earlier $(8,18,30-34)$, growth conditions critically impact on growth factor receptor activity and downstream signaling offering a certain degree of plasticity for bypass signaling. In cancer cells, these mechanisms may considerably contribute to radio- and chemoresistance as well as resistance to novel molecular agents. Our study presents compelling data on the resemblance of EGFR-associated signaling in 3D lrECM cell cultures and corresponding tumor xenografts. About the differences in EGFR phosphorylation in $3 \mathrm{D}$ versus $2 \mathrm{D}$, the similarity in the cytotoxicity profiles of cetuximab was unexpected. Importantly, radiosensitization of 3D SCC cell cultures corroborated with tumor sensitization of SCC tumor xenografts $(7,9)$ and improved locoregional control and overall survival of patients with head and neck cancer treated with combined radiotherapy and cetuximab $(11,12)$. A mechanistic explanation for cetuximab-mediated radioprotection of $2 \mathrm{D}$-grown cells is currently lacking but should take into consideration signal transduction events, gene expression, and cell cycling $(20,21,25)$.

In line with current knowledge (1), reduced phosphorylation of EGFR downstream signaling mediators such as MEK, ERK1/2, and CREB as well as PI3K/Akt at kinaseactivating amino acid residues occurred in response to cetuximab treatment. This compromised promitogenic and prosurvival signaling and translated into significant cytotoxicity and radiosensitization. Taking methodologic limitations into account, circumvention of EGFR proximal Grb2/ Ras/Raf signaling was unexpected. Instead, hyperphosphorylation/activation of Rac-MEK4/7-JNK-c-Jun signaling components, acting in a context-dependent manner, was found in 3D HNSCC cell cultures and tumor xenografts on cetuximab treatment. By channeling into S63 phosphorylation of the JNK2 downstream target c-Jun, we hypothesize JNK2-cJun activation to critically cooperate in an efficacy-limiting stress response to cetuximab.

The JNK family members serve in a multifunctional, environment-driven, and cell line/type-dependent manner $(35,36)$. Among many factors, X-ray radiation is a well-known JNK activator $(36,37)$. To prove whether JNK2 is mechanistically involved in a cetuximab-self-attenuating reaction, siRNAmediated JNK2 depletion and pharmacologic JNK inhibition (SP600125) were conducted. Although JNK2 inhibition conferred cytotoxicity and radiosensitization on its own, concomitant JNK2 knockdown plus cetuximab acted in an additive manner. By using a cetuximab-resistant cell line, that is, UTSCC5, we were able to confirm the importance of JNK2 for cetuximab-mediated radiosensitization.

\section{References}

1. Hynes NE, MacDonald G. ErbB receptors and signaling pathways in cancer. Curr Opin Cell Biol 2009;21:177-84.

2. Shepard HM, Brdlik CM, Schreiber H. Signal integration: a framework for understanding the efficacy of therapeutics targeting the human EGFR family. J Clin Invest 2008;118:3574-81.
One possible candidate connecting EGFR and JNK2 is JIP-4, which we found by mass spectrometry of cetuximab-EGFR immunoprecipitates. JIPs serve as scaffold proteins for interactions of different MAPK family members for distributing biochemical cues through specific signaling cascades $(38,39)$. By means of colocalization studies and gene silencing, we provide evidence that JIP-4 is more intensively recruited to cetuximab-inhibited, membranous EGFR and that JIP-4 depletion prevents cetuximab-mediated JNK2 and c-Jun hyperphosphorylation. Moreover, single JIP-4 knockdown provoked radiosensitization similar to cetuximab alone and, importantly, elicited higher cetuximab efficacy for radiosensitization in the combined JIP-4 knockdown/cetuximab regimen.

In summary, our findings show a high degree of similarity in EGFR downstream signaling in 3D IrECM cell cultures and corresponding tumor xenografts. Because tumor cells are thought to have multiple bypass possibilities under treatment, the presented data suggest participation of JNK2 in cetuximabantagonizing signaling pathways via interaction between EGFR and the scaffold protein JIP-4, thus providing important novel insights into tumor cell responses and resistance mechanisms to a targeted therapy that has already reached the clinic.

\section{Disclosure of Potential Conflicts of Interest \\ No potential conflicts of interest were disclosed.}

\section{Authors' Contributions}

Conception and design: I. Eke, N. Cordes

Development of methodology: I. Eke, N. Cordes

Acquisition of data (provided animals, acquired and managed patients, provided facilities, etc.): I. Eke, L. Schneider, C. Förster, D. Zips

Analysis and interpretation of data (e.g., statistical analysis, biostatistics, computational analysis): I. Eke, L. Schneider, C. Förster, D. Zips, N. Cordes Writing, review, and/or revision of the manuscript: I. Eke, L.A. KunzSchughart, N. Cordes

Administrative, technical, or material support (i.e., reporting or organizing data, constructing databases): L.A. Kunz-Schughart

Study supervision: $\mathrm{N}$. Cordes

\section{Acknowledgments}

The authors thank A. Shevchenko (Max Planck Institute of Molecular Cell Biology and Genetics, Dresden, Germany) for mass spectrometry analysis, R. Grenman (Turku University Central Hospital, Finland) for the UTSCC14 and 5 cell lines, E. Hammond for critical reading of the article, and I. Lange for excellent technical assistance.

\section{Grant Support}

This work was supported in part by the Bundesministerium für Bildung und Forschung (BMBF-03ZIK041 to N. Cordes, BMBF-02NUK006B to N. Cordes, L. Kunz-Schughart, and D. Zips), the EFRE Europäische Fonds für regionale Entwicklung, Europa fördert Sachsen (100066308), and by the Medical Faculty Carl Gustav Carus, Dresden University of Technology, Germany (MeDDrive program to I. Eke).

The costs of publication of this article were defrayed in part by the payment of page charges. This article must therefore be hereby marked advertisement in accordance with 18 U.S.C. Section 1734 solely to indicate this fact.

Received May 29, 2012; revised September 12, 2012; accepted October 3, 2012; published OnlineFirst December 27, 2012.

3. Wheeler DL, Dunn EF, Harari PM. Understanding resistance to EGFR inhibitors -impact on future treatment strategies. Nat Rev Clin Oncol 2010;7:493-507.

4. Zips D, Krause M, Yaromina A, Dorfler A, Eicheler W, Schutze C, et al. Epidermal growth factor receptor inhibitors for radiotherapy: 
biological rationale and preclinical results. J Pharm Pharmacol 2008;60:1019-28.

5. Jackman D, Pao W, Riely GJ, Engelman JA, Kris MG, Janne PA, et al. Clinical definition of acquired resistance to epidermal growth factor receptor tyrosine kinase inhibitors in non-small-cell lung cancer. J Clin Oncol 2010;28:357-60.

6. Yatabe $\mathrm{Y}$, Takahashi T, Mitsudomi T. Epidermal growth factor receptor gene amplification is acquired in association with tumor progression of EGFR-mutated lung cancer. Cancer Res 2008;68:2106-11.

7. Gurtner $K$, Deuse $Y$, Butof R, Schaal K, Eicheler W, Oertel R, et al. Diverse effects of combined radiotherapy and EGFR inhibition with antibodies or TK inhibitors on local tumour control and correlation with EGFR gene expression. Radiother Oncol 2011;99:323-30.

8. Eke I, Cordes N. Dual targeting of EGFR and focal adhesion kinase in 3D grown HNSCC cell cultures. Radiother Oncol 2011;99:279-86.

9. Krause M, Schutze C, Petersen C, Pimentel N, Hessel F, Harstrick A, et al. Different classes of EGFR inhibitors may have different potential to improve local tumour control after fractionated irradiation: a study on C225 in FaDu hSCC. Radiother Oncol 2005;74:109-15.

10. Saleh MN, Raisch KP, Stackhouse MA, Grizzle WE, Bonner JA, Mayo MS, et al. Combined modality therapy of A431 human epidermoid cancer using anti-EGFr antibody C225 and radiation. Cancer Biother Radiopharm 1999;14:451-63.

11. Bonner JA, Harari PM, Giralt J, Azarnia N, Shin DM, Cohen RB, et al. Radiotherapy plus cetuximab for squamous-cell carcinoma of the head and neck. N Engl J Med 2006;354:567-78.

12. Bonner JA, Harari PM, Giralt J, Cohen RB, Jones CU, Sur RK, et al. Radiotherapy plus cetuximab for locoregionally advanced head and neck cancer: 5-year survival data from a phase 3 randomised trial, and relation between cetuximab-induced rash and survival. Lancet Oncol 2010;11:21-8.

13. Lu Y, Li X, Liang K, Luwor R, Siddik ZH, Mills GB, et al. Epidermal growth factor receptor (EGFR) ubiquitination as a mechanism of acquired resistance escaping treatment by the anti-EGFR monoclonal antibody cetuximab. Cancer Res 2007;67:8240-7.

14. Wheeler DL, lida M, Kruser TJ, Nechrebecki MM, Dunn EF, Armstrong EA, et al. Epidermal growth factor receptor cooperates with Src family kinases in acquired resistance to cetuximab. Cancer Biol Ther 2009;8: 696-703.

15. Huang S, Benavente S, Armstrong EA, Li C, Wheeler DL, Harari PM. p53 modulates acquired resistance to EGFR inhibitors and radiation. Cancer Res 2011;71:7071-9.

16. Yonesaka K, Zejnullahu K, Okamoto I, Satoh T, Cappuzzo F, Souglakos J, et al. Activation of ERBB2 signaling causes resistance to the EGFR-directed therapeutic antibody cetuximab. Sci Transl Med 2011;3:99ra86.

17. Eke I, Leonhardt F, Storch K, Hehlgans S, Cordes N. The small molecule inhibitor QLT0267 radiosensitizes squamous cell carcinoma cells of the head and neck. PLoS ONE 2009;4:e6434.

18. Kenny PA. Three-dimensional extracellular matrix culture models of EGFR signalling and drug response. Biochem Soc Trans 2007;35: 665-8.

19. Le Beyec J, Xu R, Lee SY, Nelson CM, Rizki A, Alcaraz J, et al. Cell shape regulates global histone acetylation in human mammary epithelial cells. Exp Cell Res 2007;313:3066-75.

20. Nelson CM, Inman JL, Bissell MJ. Three-dimensional lithographically defined organotypic tissue arrays for quantitative analysis of morphogenesis and neoplastic progression. Nat Protoc 2008;3:674-8.

21. Pampaloni $F$, Reynaud EG, Stelzer EH. The third dimension bridges the gap between cell culture and live tissue. Nat Rev Mol Cell Biol 2007;8:839-45.
22. Storch K, Eke I, Borgmann $\mathrm{K}$, Krause $\mathrm{M}$, Richter $\mathrm{C}$, Becker $\mathrm{K}$, et al. Three-dimensional cell growth confers radioresistance by chromatin density modification. Cancer Res 2010;70:3925-34.

23. Weaver VM, Lelievre S, Lakins JN, Chrenek MA, Jones JC, Giancotti F, et al. beta4 Integrin-dependent formation of polarized three-dimensional architecture confers resistance to apoptosis in normal and malignant mammary epithelium. Cancer Cell 2002;2:205-16.

24. Martin KJ, Patrick DR, Bissell MJ, Fournier MV. Prognostic breast cancer signature identified from $3 \mathrm{D}$ culture model accurately predicts clinical outcome across independent datasets. PLOS ONE 2008;3: e2994.

25. Zschenker O, Streichert T, Hehlgans S, Cordes N. Genome-wide gene expression analysis in cancer cells reveals $3 \mathrm{D}$ growth to affect $\mathrm{ECM}$ and processes associated with cell adhesion but not DNA repair. PLoS ONE 2012;7:e34279.

26. Eke I, Deuse Y, Hehlgans S, Gurtner K, Krause M, Baumann M, et al. $\beta 1$ Integrin/FAK/cortactin signaling is essential for human head and neck cancer resistance to radiotherapy. J Clin Invest 2012;122:1529-40.

27. Eke I, Koch U, Hehlgans S, Sandfort V, Stanchi F, Zips D, et al. PINCH regulates Akt1 activation and enhances radioresistance by inhibiting PP1alpha. J Clin Invest 2010;120:2516-27.

28. Yan H, Zhang B, Li S, Zhao Q. A formal model for analyzing drug combination effects and its application in TNF-alpha-induced NFkappaB pathway. BMC Syst Biol 2010;4:50.

29. Shevchenko A, Roguev A, Schaft D, Buchanan L, Habermann B, Sakalar C, et al. Chromatin central: towards the comparative proteome by accurate mapping of the yeast proteomic environment. Genome Biol 2008;9:R167.

30. Roskelley CD, Desprez PY, Bissell MJ. Extracellular matrix-dependent tissue-specific gene expression in mammary epithelial cells requires both physical and biochemical signal transduction. Proc Natl Acad Sci U S A 1994;91:12378-82.

31. Wang F, Weaver VM, Petersen OW, Larabell CA, Dedhar S, Briand P, et al. Reciprocal interactions between beta1-integrin and epidermal growth factor receptor in three-dimensional basement membrane breast cultures: a different perspective in epithelial biology. Proc Nat Acad Sci U S A 1998;95:14821-6.

32. Eke I, SandfortV, Mischkus A, Baumann M, Cordes N. Antiproliferative effects of EGFR tyrosine kinase inhibition and radiation-induced genotoxic injury are attenuated by adhesion to fibronectin. Radiother Oncol 2006;80:178-84.

33. Eke I, Sandfort V, Storch K, Baumann M, Roper B, Cordes N. Pharmacological inhibition of EGFR tyrosine kinase affects ILK-mediated cellular radiosensitization in vitro. Int J Radiat Biol 2007:83:793-802.

34. Hehlgans S, Lange I, Eke I, Cordes N. 3D cell cultures of human head and neck squamous cell carcinoma cells are radiosensitized by the focal adhesion kinase inhibitor TAE226. Radiother Oncol 2009;92: 371-8.

35. Hochedlinger K, Wagner EF, Sabapathy K. Differential effects of JNK1 and JNK2 on signal specific induction of apoptosis. Oncogene 2002;21:2441-5.

36. Weston CR, Davis RJ. The JNK signal transduction pathway. Curr Opin Cell Biol 2007;19:142-9.

37. Cordes N, Seidler J, Durzok R, Geinitz H, Brakebusch C. Beta1integrin-mediated signaling essentially contributes to cell survival after radiation-induced genotoxic injury. Oncogene 2006;25:1378-90.

38. Lee CM, Onesime D, Reddy CD, Dhanasekaran N, Reddy EP. JLP: a scaffolding protein that tethers JNK/p38MAPK signaling modules and transcription factors. Proc Natl Acad Sci U S A 2002;99:14189-94.

39. Whitmarsh AJ. The JIP family of MAPK scaffold proteins. Biochem Soc Trans 2006;34:828-32. 


\section{Cancer Research}

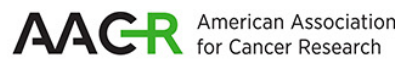

The Journal of Cancer Research (1916-1930) | The American Journal of Cancer (1931-1940)

\section{EGFR/JIP-4/JNK2 Signaling Attenuates Cetuximab-Mediated Radiosensitization of Squamous Cell Carcinoma Cells}

Iris Eke, Lydia Schneider, Claudia Förster, et al.

Cancer Res 2013;73:297-306. Published OnlineFirst October 16, 2012.

Updated version Access the most recent version of this article at: doi:10.1158/0008-5472.CAN-12-2021

Supplementary Access the most recent supplemental material at:

Material http://cancerres.aacrjournals.org/content/suppl/2012/10/17/0008-5472.CAN-12-2021.DC1

Cited articles This article cites 39 articles, 9 of which you can access for free at:

http://cancerres.aacrjournals.org/content/73/1/297.full\#ref-list-1

Citing articles This article has been cited by 2 HighWire-hosted articles. Access the articles at: http://cancerres.aacrjournals.org/content/73/1/297.full\#related-urls

E-mail alerts Sign up to receive free email-alerts related to this article or journal.

Reprints and To order reprints of this article or to subscribe to the journal, contact the AACR Publications Department at Subscriptions pubs@aacr.org.

Permissions To request permission to re-use all or part of this article, contact the AACR Publications Department at permissions@aacr.org. 